\title{
CORRIGENDUM
}

\section{A dopamine transporter mutation associated with bipolar affective disorder causes inhibition of transporter cell surface expression}

\author{
S Horschitz, R Hummerich, T Lau, M Rietschel and P Schloss
}

Molecular Psychiatry (2006) 11, 704. doi:10.1038/sj.mp.4001849

Correction to: Molecular psychiatry (2005) 10, 11041109. doi: 10.1038/sj.mp.4001730

Two rare missense substitutions (A559V and E602G) of the human dopamine transporter (hDAT) have been identified. The characterization of these mutants in a heterologous cellular system stably expressing the respective transporter proteins was described in Molecular Psychiatry (2005) 10, 1104-1109. Whereas the properties of the A559V mutant protein were comparable to wild-type hDAT, clones stably expressing the E602G mutant showed no substrate transport nor antagonist binding. Confocal microscopy revealed that in these cells the E602G protein is expressed but not delivered to the cell surface.

Mazei-Robison and Blakely have published in Neuropharmacology (2005) 49, 737-749, a characterization of several naturally occurring mutants of hDAT. In contrast to our data, in their study the
E602G mutant was fully active and comparable to wild-type hDAT. In order to elucidate these contradictory results, both our labs exchanged the mutant cDNAs and re-analysed the properties of the E602G mutant. Uptake experiments in both labs with HEK 293 transiently transfected with both cDNA plasmids revealed transport activity of the E602G mutant comparable to wild-type hDAT. Thus, our conclusion published in Molecular Psychiatry (2005) 10, 1104-1109 is wrong and has to be withdrawn at this point of time.

Mazei and Blakely have also engineered several stable E602G lines and found these to express equivalent amounts of protein/transport, suggesting it is not the production of stable lines per se that explains the results obtained with our stable lines. Reanalyses of our clones expressing the E602 mutant were still consistent with our published data. Currently, we have no explanation for the observed properties of these cell lines. 